\title{
Disección coronaria espontánea asociada a lupus eritematoso sistémico
}

\section{Spontaneous coronary dissection associated with systemic lupus erythematosus}

\author{
Manuel García-Sánchez ${ }^{1 *}$, Jorge Carrillo², Yuyi Montero ${ }^{3}$ y Daniel Seniscal4 \\ ${ }^{1}$ Servicio de Cardiología Clínica, Universidad Nacional Autónoma de México (UNAM); ${ }^{2}$ Servicio de Cardiología Intervencionista, Unidad de \\ Hemodinámica Cardiovascular; ${ }^{3}$ Servicio de Cardiología y Ecocardiografía, Unidad de Cuidados Intensivos Coronarios. Centro Médico Nacional \\ La Raza Dr. Antonio Fraga Mouret; ${ }^{4}$ Servicio de Medicina Interna, Centro Médico ABC, UNAM. Ciudad de México, México
}

\begin{abstract}
Resumen
A 29 year old female with a past medical history of systemic lupus erythematosus, diagnosed 15 years earlier, presents with lupus nephritis, currently on peritoneal dialysis. She had myopericarditis in 2012 and is currently on immunosuppressants. The patient began with exertional dyspnea and angina 2 weeks before admission. An echocardiogram was performed, reporting severe mitral and tricuspid insufficiency. Afterwards, the patient presented with resting angina associated with an adrenergic and vagal response. Initially, rheumatology ruled out autoimmune activity caused by lupus. We performed a coronary angiogram based on clinical presentation, EKG changes and biomarkers, finding a trivascular coronary artery disease classified as a Markis I coronary artery ectasia and a coronary dissection of the ramus intermedius and the circumflex, posterior to the first obtuse marginal artery. Cardiothoracic surgery considered intervention with a coronary bridge posterior to the dissection of the intermedius ramus artery, marginal obtuse and posterolateral artery, as well as a mitral valve replacement and a tricuspid valve repair. Coronary dissection is more common in women (70\%), clinical presentation varies from unstable angina to sudden death. In lupus nephritis, it is an uncommon form of extra renal vasculitis. Treatment depends on the number of arteries affected, as well as the haemodynamic state of the patient. It is imperative to individualize treatment options.
\end{abstract}

Palabras clave: Disección coronaria. Ectasia coronaria. Nefropatía lúpica. Vasculitis.

\begin{abstract}
Se presenta el caso de una paciente de 29 años con antecedente de lupus eritematoso sistémico diagnosticado 15 años antes, que desarrolló nefropatía lúpica actualmente en diálisis peritoneal, cuadro de miopericarditis en 2012 y bajo tratamiento inmunosupresor. Inició con deterioro de su clase funcional por disnea y angina 2 semanas previas al ingreso. Se le realizó ecocardiograma, el cual reportó insuficiencias mitral y tricuspídea graves. Posteriormente presentó angina en reposo asociada a descarga adrenérgica y vagal. A su ingreso se descarta actividad lúpica por reumatología. Por presentación clínica, cambios en electrocardiograma y biomarcadores, se realizó cateterismo cardiaco, que reportó enfermedad coronaria trivascular con ectasia coronaria Markis I y disección coronaria de ramo intermedio y circunfleja posterior a la primera marginal obtusa. Se consideró por el servicio de cirugía cardiotorácica realizar intervención con puente coronario posterior a
\end{abstract}

Correspondencia:

*Manuel I. García-Sánchez E-mail: meny.nem@gmail.com
Disponible en internet: 23-11-2020 Arch Cardiol Mex. 2021;91(1):114-120 www.archivoscardiologia.com 1405-9940 / @ 2020 Instituto Nacional de Cardiología Ignacio Chávez. Publicado por Permanyer. Este es un artículo open access bajo la licencia CC BY-NC-ND (http://creativecommons.org/licenses/by-nc-nd/4.0/). 
disección del ramo intermedio, marginal obtusa y posterolateral, así como cambio valvular mitral y plastia tricuspídea. La disección coronaria espontánea es más frecuente en las mujeres (70\%); puede presentarse como angina inestable y hasta como muerte súbita. La asociación con lupus eritematoso sistémico es poco frecuente, con una incidencia del $0.42 \%$. En la nefropatía lúpica es una manifestación poco frecuente de vasculitis extrarrenal. El tratamiento de elección depende del número de vasos afectados y del estado hemodinámico, por lo que es necesario individualizarlo para cada paciente.

Key words: Coronary dissection. Coronay ectasia. Lupus nephritis. Vasculitis.

\section{Introducción}

La disección espontánea de las arterias coronarias (SCAD, spontaneous coronary artery dissection) es un diagnóstico de exclusión poco frecuente en los síndromes coronarios agudos, con un prevalencia reportada del $0.1-1.1 \%$, y la mayoría de los reportes son en personas menores de 50 años y mujeres en periodo periparto $^{1,2}$. Se define como una separación espóntanea de la pared de una arteria coronaria que no es iatrogénica ni por trauma, y el término excluye la etiología ateroesclerosa ${ }^{3}$. Actualmente se aceptan dos hipotesis en la patogenia: el desgarro de la íntima con la formación subsecuente de un hematoma intramural y la hemorragia de la capa media por lesión en los vasa vasorum $^{3,4}$. El debut clínico varía desde el síndrome coronario agudo en todas sus variantes, la insuficiencia cardiaca hasta incluso la muerte súbita ${ }^{4}$. Por lo general, existe una arteriopatía predisponente de trasfondo y un estresor desencadenante. En el primer caso se incluye la displasia fibromuscular (asociación más dominante), en relación con el embarazo, enfermedades del tejido conectivo, enfermedades inflamatorias sistémicas, terapia hormonal o idiopático. Respecto a los estresores predisponentes, destacan el estrés y el ejercicio intensos, el parto, las actividades con Valsalva intenso y algunas drogas adictivas, entre otros. En reportes de casos, las enfermedades inflamatorias autoinmunitarias se han asociado a SCAD en menos del $1 \%$ de los casos, aunque probablemente esté infraestimado; sin embargo, como factor predisponente se ha propuesto la inflamación crónica por vasculitis ${ }^{4,5}$.

Existen diferencias en cuanto a la presentación, la evolución y el pronóstico en SCAD de etiología ateroesclerosa y no ateroesclerosa, principalmente por el tratamiento que conlleva esta última, por lo cual se requiere un diagnóstico temprano y preciso ${ }^{6}$. La angiografía coronaria ha sido el método de primera línea en el diagnóstico de SCAD, pero debido a las limitaciones en la valoración de los fotogramas por segundo y la falta de mayor definición estructural del vaso en cuestión, hoy en día se cuenta con la posibilidad de realizar durante la angiografia invasiva la toma de imágenes intracoronarias por medio de ultrasonido (IVUS, intravascular ultrasound) y de tomografía de coherencia óptica (OCT, optical coherence tomography), que complementan el diagnóstico y el manejo de la SCAD?

Las modalidades terapéuticas se han propuesto basándose en numerosas series de casos. Idealmente, la estratificación del riesgo según los hallazgos clínicos y de imagen apoya el manejo individualizado, desde conservador hasta cirugía con puenteo aortocoronario ${ }^{3,7}$.

Presentamos el caso de una mujer joven con lupus eritematoso sistémico que se presenta con angina inestable e insuficiencia cardiaca descompensada, en quien inicialmente se sospechó actividad lúpica como causa de la descompensación y en su evolución intrahospitalaria se produjo el hallazgo angiográfico de disección coronaria espontánea en más de un vaso.

\section{Presentación del caso}

Mujer de 29 años con antecedente de lupus eritematoso sistémico en tratamiento con prednisona, nefropatía lúpica en terapia de reemplazo renal con diálisis peritoneal y antecedente de miopericarditis aguda en 2012.

Inició con deterioro de su clase funcional por disnea y angina 2 semanas antes de su ingreso, siendo progresiva de grandes a pequeños esfuerzos. En el ecocardiograma inicial se concluyó insuficiencia mitral y tricuspídea grave, dilatación biauricular sin trombos, con disfunción diastólica de grado II, fracción de eyección del ventrículo izquierdo del 35\%, con hipocinesia generalizada, y función sistólica del ventrículo derecho conservada (Fig. 1). A su ingreso, el servicio de reumatología descartó actividad lúpica. Posteriormente, durante la hospitalización, presentó un episodio de angina en reposo asociada a descarga adrenérgica, empeorando la disnea. Se realizó un electrocardiograma durante el episodio que mostró ritmo sinusal, intervalo PR y QT en rango normal, datos de hipertrofia septal y ventrículo izquierdo con ondas $T$ positivas acuminadas simétricas en las derivaciones precordiales. Los biomarcadores específicos mostraron una troponina I 


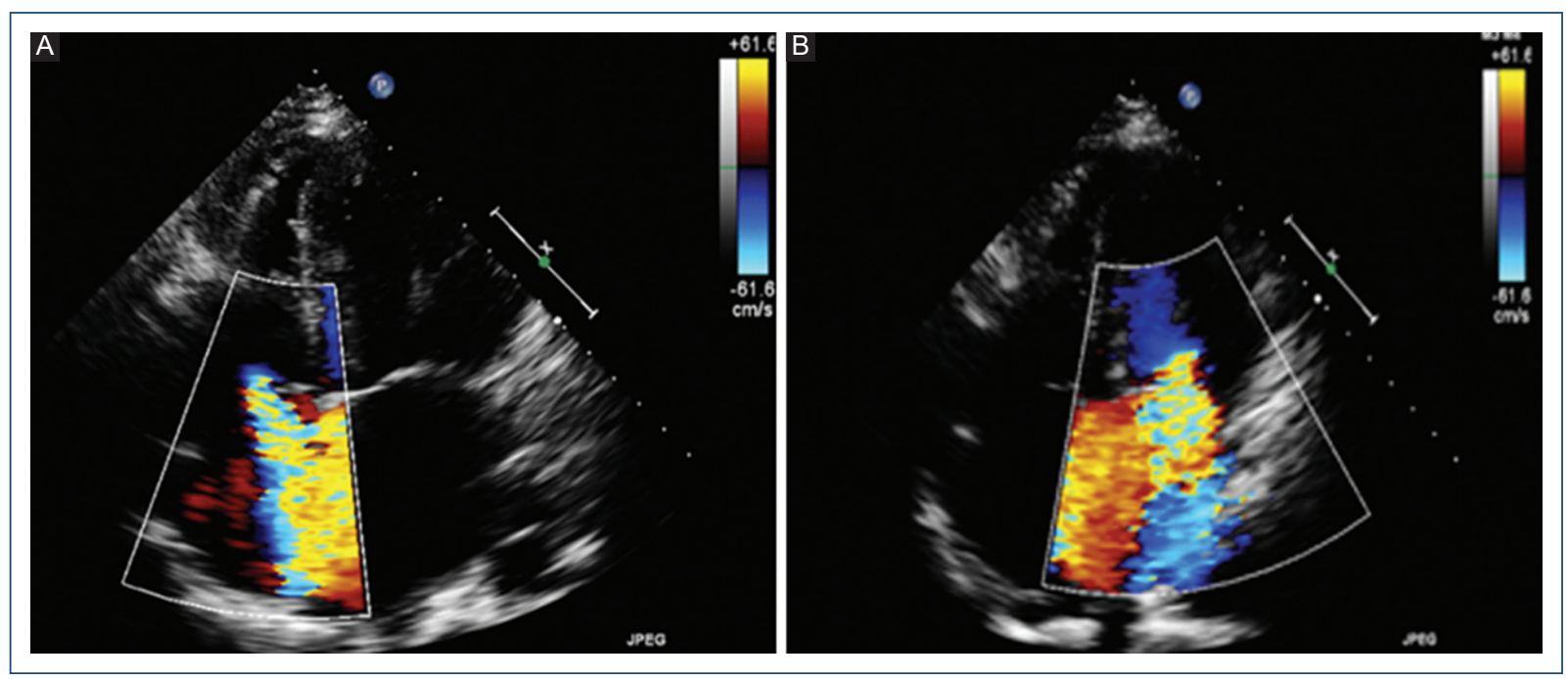

Figura 1. A: ecocardiograma, imagen apical de cuatro cámaras, que muestra insuficiencia tricuspídea grave con vena contracta de $10.2 \mathrm{~mm}$. El área de la aurícula derecha es de $21 \mathrm{~cm}^{2}$. B: ecocardiograma, imagen apical de cuatro cámaras, que muestra insuficiencia mitral grave con un área del orificio regurgitante de $0.4 \mathrm{~cm}^{2}$. El volumen regurgitante es de $125 \mathrm{ml}$ y en la aurícula izquierda $42 \mathrm{ml} / \mathrm{m}^{2}$.

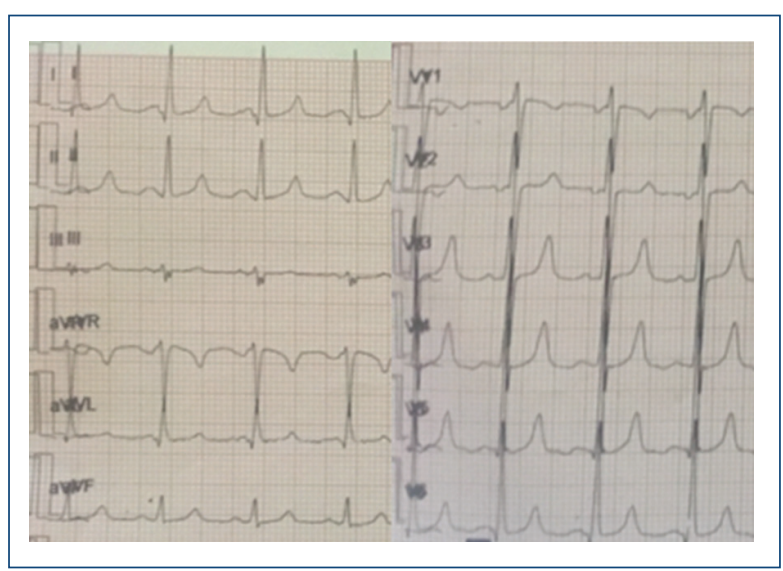

Figura 2. Electrocardiograma de 12 derivaciones que muestra ritmo sinusal, eje QRS $10^{\circ}$, onda P $140 \mathrm{~ms}, \mathrm{PR}$ $200 \mathrm{~ms}$, QRS $100 \mathrm{~ms}$, voltaje aumentado en precordiales, Cornell $25 \mathrm{~mm}$ y ondas T simétricas acuminadas de $10 \mathrm{~mm}$, sugestivas de isquemia subendocárdica.

de $0.24 \mathrm{ng} / \mathrm{ml}$, generando la sospecha de un trastorno isquémico subyacente (Fig. 2), por lo cual fue valorada por el servicio de cardiología intervencionista hemodinámica, que justificó la realización de un cateterismo cardiaco en el que se reportó enfermedad coronaria trivascular con ectasia coronaria Markis I, disección coronaria de ramo intermedio y circunfleja distal a primera marginal obtusa (Fig. 3). Se consideró por el servicio de cirugía cardiotorácica la realización de cirugía con puentes coronarios posterior a disección del ramo intermedio, marginal obtuso y posterolateral, así como cambio valvular mitral y plastia tricuspídea.

\section{Discusión del caso}

La SCAD fue descrita por primera vez en 1931 en la autopsia de una mujer de 42 años, y desde entonces se han realizado múltiples reportes de caso y revisiones cortas al respecto, pero aún no se cuenta con guías clínicas específicas ${ }^{7-9}$. La SCAD consiste en la separación de dos de las tres capas de la pared arterial para crear una luz falsa, generalmente entre la media y la íntima. Es más frecuente en las mujeres (70\%), incluso en relación con el embarazo $(30 \%$ de los casos $)^{10}$, y se presenta como angina $(95.9 \%)$ o incluso como muerte súbita $(<0.5 \%)^{10-13}$. Su asociación con lupus eritematoso sistémico es poco frecuente, con una incidencia del $0.42 \%$, siendo el riesgo tres veces mayor que en la población normal ${ }^{12}$. Desde el punto de vista de la nefropatía lúpica, es una manifestación poco frecuente de vasculitis extrarrenal. De forma clásica se consideraba la etiología de la SCAD como posparto o periparto, ateroesclerosa e idiopática; sin embargo, con el desarrollo de nuevos métodos de imágen se ha descrito su asociación con los síndromes de Ehlers-Danlos y de Marfan, la fibrodisplasia, el uso de cocaína o de anticonceptivos orales y el ejercicio extremo. Con el fin de englobar la etiología, Saw, et al. ${ }^{3}$ proponen cuatro 

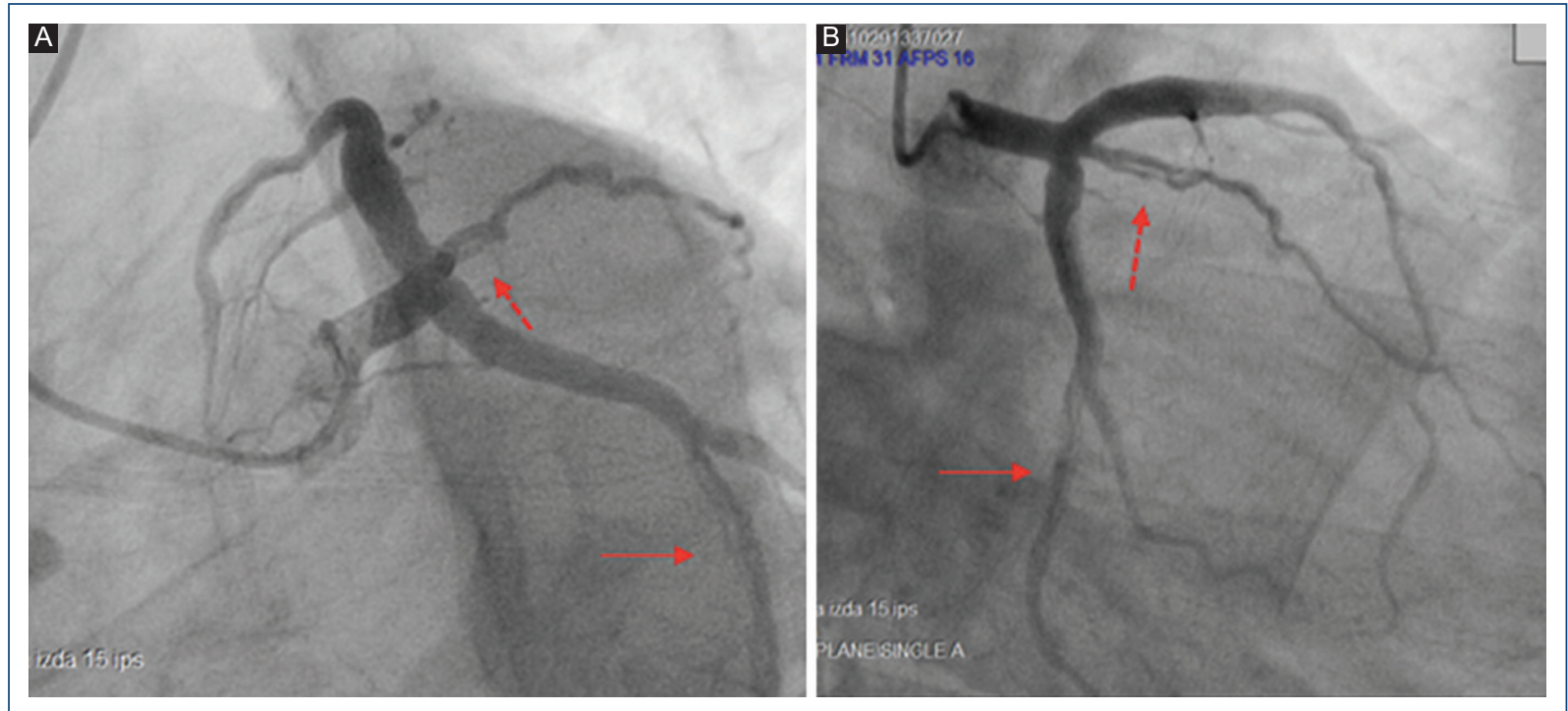

Figura 3. A: angiografía coronaria, proyección oblicua anterior izquierda caudal, que muestra una disección de tipo B del NHLBI o de tipo 1 de Saw del segmento proximal del ramo intermedio (flecha ancha); disección de tipo B del segmento distal de la arteria circunfleja (flecha delgada). B: angiografía coronaria, proyección oblicua anterior izquierda craneal, que muestra una disección de tipo B del NHLBI o de tipo 1 de Saw del segmento proximal del ramo intermedio (flecha ancha) y una disección de tipo B del segmento distal de la arteria circunfleja, con mayor extensión (flecha delgada).

grupos: 1) asociada a enfermedades del tejido conectivo, 2) pacientes con ateroesclerosis o vasculitis, 3) periparto y 4) idiopática ${ }^{3,12-14}$.

El diagnóstico inicial se realiza mediante angiografía coronaria invasiva, la cual continúa siendo el método de primera línea; no obstante, debido a sus limitaciones en la caracterización de las lesiones y la subsecuente estratificación del riesgo, las técnicas de imagen intracoronaria, como IVUS y OCT, se han propuesto en el algoritmo de diagnóstico y tratamiento. Sin embargo, aunque son estudios ideales, no son esenciales en la práctica clínica, ya que no todos los centros cuentan con estos métodos de imagen invasivos. Por lo tanto, la identificación inicial por angiografia coronaria toma protagonismo. Previamente, el National Heart, Lung, and Blood Institute (NHLBI) estableció seis tipos de disección coronaria (A-F). En el caso de esta paciente consideramos que se trataba del tipo $B$ debido al doble lumen separado por un área de radiotransparencia, con persistencia después del lavado de contraste. Sin embargo, debido al solapamiento de criterios con otras afecciones cuando se trata de los tipos $\mathrm{C}$ a F, Saw, et al. ${ }^{3}$, basándose en las numerosas caracterizaciones por imagen intracoronaria, proponen en su revisión tres tipos en la clasificación angiográfica: 1) la lesión patognomónica es la tinción con contraste de la pared arterial con múltiples lúmenes radiolúcidos, y ocurre en el 29\% de casos; 2) lesiones difusas, típicamente $>30$ $\mathrm{mm}$, sin un plano de disección visible, que pueden resultar en oclusión completa del vaso y a su vez se clasifican en dos subtipos, A (lesiones segmentarias) y B (lesiones extendidas en todo el vaso), siendo el tipo B el más frecuente (67.5\%); y 3) lesiones focales 0 tubulares que sugieren estenosis por ateroesclerosis y en general requieren estudios de imagen intracoronaria para confirmar el diagnóstico, representando el 3.4\% de los $\operatorname{casos}^{3,14}$. En cuanto al predominio del vaso afectado, la arteria descendente anterior es la más afectada (34-42\%), comprometiendo los segmentos medios a distales, y en menos del $10 \%$ de los casos los segmentos proximales. La disección de múltiples vasos se presenta en el 9-19\% de los casos ${ }^{4,5,15}$. Sin embargo, estos últimos porcentajes se obtienen de revisiones que incluyen todas las etiologías de SCAD.

Existen varios algoritmos de manejo y, en este rubro, los mayores expositores del tema, Saw, et al. ${ }^{3}$, refieren el diagnóstico escalonado iniciando con angiografía coronaria, y según la presentación clínica mencionada previamente se debe buscar de manera objetiva la apariencia tipo 1 de SCAD. En caso de no presentarse esta, se buscará identificar estenosis fina y difusa como la descrito en la SCAD tipo 2; en este 
paso se sugiere la administración intravenosa de nitroglicerina para realizar IVUS u OCT con el objetivo de definir la posibilidad de revalorar en 4 a 6 semanas. Por otro lado, si no se cuenta con esas características, se buscará la apariencia de tipo 3 , la cual simula lesiones por ateroesclerosis, y en esta última será imprescindible realizar estudios de imagen intracoronarios $^{3,4,7,15}$. Sobre los modos de imagen, la OCT tiene la mejor resolución para definir rotura de la íntima, trombo y hematoma intramural, y falso lumen; sin embargo, debido a la menor penetrancia, tiene mayor dificultad para definir la extensión de algunos hematomas intramurales. Aunque existen evidentes ventajas, hay que considerar los riesgos de una intervención coronaria, como extender la disección de forma mecánica con el catéter o de forma hidráulica con el contraste, e incluso el riesgo de trombosis que conlleva introducir el catéter ${ }^{16}$. La angiotomografía no se incluye en las decisiones debido a la limitación espacial para identificar lesiones en vasos distales (zona de afección más frecuente) ${ }^{16,17}$. Respecto a la paciente del caso clínico, la interpretación que podemos realizar de las lesiones podría orientarnos hacia la apariencia clínica común del tipo 1, con doble lumen observado en la arteria circunfleja y el ramo intermedio. La paciente también inició el cuadro clínico con angina inestable y descompensación de insuficiencia cardiaca, ambos factores de alto riesgo de complicaciones cardiovasculares. Del mismo modo, otro factor clave en cuanto a las posibilidades de tratamiento es la ectasia coronaria Markis I, que definitivamente modifica la posibilidad de intervenciones percutáneas y nos hace recordar las bastas consideraciones a tener en cuenta para el tratamiento farmacológico y las implicaciones hemodinamicas del flujo en la revascularización de un vaso ectásico.

Actualmente aún no está establecido el tratamiento óptimo, ya que no existen ensayos clínicos aleatorizados sobre el manejo conservador en comparación con las estrategias de revascularización, y la evidencia se basa en series de casos, en los que la etiología principal de la SCAD es la ateroesclerosis, por lo cual, en el caso de nuestra paciente, es necesario individualizar el manejo porque, aunque no se descarta un trasfondo aterogénico, la presentación clínica orienta a distintas vías fisiopatológicas. Es de conocimiento que la trombólisis constituye un riesgo elevado por la posibilidad de aumentar el hematoma y la disección ${ }^{15,17}$. El manejo conservador se ha propuesto en pacientes estables de bajo riesgo de complicaciones y progresión, basándose en revisiones que mencionan una resolución espontánea en 4-6 semanas en casos no complejos ${ }^{3,15-18}$. El intervencionismo coronario percutáneo sigue siendo la primera opción de manejo invasivo, aunque se han descrito complicaciones hasta en el $53 \%$ de los casos, requiriendo revascularización quirúrgica ${ }^{4,15,17}$. Así mismo, el mayor número de vasos comprometidos se inclina por el manejo quirúrgico. El tratamiento médico se puede considerar en caso de observar un adecuado flujo en la coronariografia posterior al cuadro agudo ${ }^{14-18}$. Debido a esta discrepancia, Saw, et al. ${ }^{3}$ proponen un algoritmo de tratamiento dicotomizado según el riesgo inicial (alto riesgo: infarto de miocardio agudo o previo, evento vascular cerebral agudo o previo, enfermedad coronaria estable, enfermedad arterial periférica y fibrilación auricular). En caso de no presentar factores de riesgo se opta por un manejo conservador con terapia médica (bloqueadores beta, antiagregantes plaquetarios, bloqueadores del eje renina-angiotensina, etc.). Se considera como alto riesgo si hay uno o más factores, y posteriormente se considera la presentacion clínica (disección de tronco coronario, angina o isquemia identificadas en curso, fibrilación o taquicardia ventricular, choque cardiogénico); si existe lesión del tronco aislada se opta por intervencionismo coronario percutáneo, y si se añaden lesiones proximales en otros vasos se considera la cirugía de revascularización. Esta última también se considera en caso de fibrilación o de taquicardia ventricular estables hemodinámicamente, 0 de angina 0 isquemia en curso identificadas en más de dos territorios vasculares en los que por algún motivo no es posible realizar intervencionismo coronario percutáneo. Por otro lado, si se presenta inestabilidad hemodinámica, ya sea en presencia de arritmias ventriculares o en caso de choque cardiogénico, es necesario mejorar el estado hemodinámico por medio de inotropismo o asistencia mecánica ventricular, entre otros $^{3,18,19}$.

En el caso de nuestra paciente, debido a su comorbilidad y antecedentes se debe considerar la directa clasificación en alto riesgo; se conoce a la paciente con disminución de la fracción de eyección, sin presentar choque cardiogénico ni arritmias ventriculares durante la hospitalización. Por angiografía coronaria se conoce la disección proximal de ramo intermedio y arteria circunfleja, aunque con dificultad para realizar implante de stent por intervencionismo coronario percutáneo debido a la presencia de ectasia coronaria Markis I. Por todo ello, según el algoritmo se justificó la realización de cirugía de revascularización. 


\section{Conclusiones}

Se trata de una mujer joven que presentaba diversas complicaciones crónicas de una enfermedad autoinmunitaria. Por los hallazgos mencionados, se sospechó un síndrome coronario agudo en el momento de la angina y la descompensación de su insuficiencia cardiaca crónica, así como por los datos del electrocardiograma y de los biomarcadores, y ulteriormente se corroboró el cuadro por angiografía coronaria. Es un caso con un análisis retrospectivo, en el que el hallazgo angiográfico se presentó por el seguimiento protocolario del dolor precordial, y con la interpretación de mínimos cambios en una herramienta diagnóstica reproducible y afín a todo cardiólogo, como es el electrocardiograma. Es de destacar que los cambios agudos de isquemia reportados en el electrocardiograma se correlacionaron con el mejor pronóstico en el periodo posquirúrgico, ya que la identificación del síndrome coronario agudo con la falta de progresión a infarto como tal permitió una mejor adaptación por los mecanismos de compensación. Como se mencionó, el manejo propuesto consideró la patología valvular encontrada, el número de vasos comprometidos y la comorbilidad crónica de la paciente, tomando en cuenta el alto riesgo de complicaciones posquirúrgicas por el requerimiento de inmunosupresión ante el riesgo de una agudización del lupus eritematoso sistémico. Durante el tiempo posquirúrgico se logró la extubación a las 36 horas. En su última valoración, la paciente se encuentra en clase funcional II de la New York Heart Association, sin angina, bajo anticoagulación y en seguimiento periódico por el equipo multidisciplinario.

A pesar de los riesgos tan altos de complicaciones quirúrgicas, la falta de mejoría con tratamiento conservador de forma inicial, así como el probable riesgo de progresión a choque cardiogénico, se consideró que la intervención quirúrgica era el paso siguiente del manejo. Es muy probable que la edad y los mecanismos de compensación orgánica ofrecieran un pronóstico favorable en la cirugía de revascularización.

La SCAD es un diagnóstico que debe considerarse siempre en mujeres jóvenes con falla cardiaca o con síndrome coronario agudo. A pesar de la falta de evidencia contundente, el juicio clínico siempre debe guiar el manejo terapéutico, y con mayor importancia cuando se individualiza el tratamiento.

\section{Conflicto de intereses}

Ninguno.

\section{Financiamiento}

La presente investigación no ha recibido ninguna beca específica de agencias de los sectores público, comercial o sin ánimo de lucro.

\section{Agradecimientos}

Para la elaboración del presente documento se contó con los recursos materiales del Instituto Mexicano del Seguro Social. Los autores expresan su agradecimiento a las autoridades del Departamento de Cardiología, a la Unidad de Cuidados Intensivos Coronarios y al Servicio de Hemodinámica del Centro Médico Nacional La Raza, por permitir el uso de los recursos materiales y personales para poder llegar al diagnóstico y el tratamiento correctos de los pacientes.

\section{Responsabilidades éticas}

Protección de personas y animales. Los autores declaran que para esta investigación no se han realizado experimentos en seres humanos ni en animales.

Confidencialidad de los datos. Los autores declaran que han seguido los protocolos de su centro de trabajo sobre la publicación de datos de pacientes.

Derecho a la privacidad y consentimiento informado. Los autores declaran que en este artículo no aparecen datos de pacientes.

\section{Bibliografía}

1. Mortensen KH, Thuesen L, Kristensen IB, Christiansen EH. Spontaneous coronary artery dissection: a Western Denmark Heart Registry study. Catheter Cardiovasc Interv. 2009;74:710-7.

2. Hering D, Piper C, Hohmann C, Schultheiss HP, Horstkotte D. [Prospective study of the incidence, pathogenesis and therapy of spontaneous, by coronary angiography diagnosed coronary artery dissection.] Z Kardiol. 1998:87:961-70.

3. Saw J, Mancini GBJ, Humphries KH. Contemporary review on spontaneous coronary artery dissection. J Am Coll Cardiol. 2016;68:297 -312. [Published correction appears in J Am Coll Cardiol. 2016; 68:1606.]

4. Saw J, Aymong E, Sedlak T, Buller CE, Starovoytov A, Ricci D, et al. Spontaneous coronary artery dissection: association with predisposing arteriopathies and precipitating stressors and cardiovascular outcomes. Circ Cardiovasc Interv. 2014:7:645-55.

5. Kamran M, Guptan A, Bogal M. Spontaneous coronary artery dissection: case series and review. J Invasive Cardiol. 2008;20:553-9.

6. Franco C, Starovoytov A, Mancini GB, et al. Spontaneous resolution of LV dysfunction post SCAD: a case series. Paper presented at: SCAI 2015, May 69, 2015; San Diego, CA.

7. Saw J. Coronary angiogram classification of spontaneous coronary artery dissection. Catheter Cardiovasc Interv. 2014;84:1115-22.

8. Pretty HC. Dissecting aneurysm of coronary artery in a woman aged 42 : rupture. BMJ. 1931;1:667.

9. Tweet MS, Hayes SN, Pitta SR, Simari RD, Lerman A, Lennon RJ, et al. Clinical features, management, and prognosis of SCAD. Circulation. 2012;126:579-88.

10. Romero-Rodríguez N, Fernández-Quero M, Villa Gil-Ortega M, Urbano del Moral JA, Ballesteros Prada S, Díaz de la Llera L, et al. Disección coronaria espontánea y sus implicaciones pronósticas a largo plazo en una cohorte de 19 casos. Rev Esp Cardiol. 2010;63:1088-91. 
Arch Cardiol Mex. 2021;91(1)

11. Shamloo BK, Chintala R, Freedman AN, Potosky A, Malin J, Weiss Smith S. Spontaneous coronary artery dissection: aggressive vs conser vative therapy. J Invasive Cardiol. 2010;22:222-8.

12. DeMaio SJ Jr, Kinsella SH, Silverman ME. Clinical course and long-term prognosis of spontaneous coronary artery dissection. Am J Cardiol. 1989;64:471-4

13. Álvarez-Lario B, Álvarez-Roy L, Mayordomo-Gómez S, García-García JM. Spontaneous coronary artery dissection in systemic lupus erythematosus: case-based review. Rheumatol Int. 2019;39:1821-7.

14. Aziz S. Spontaneous coronary artery dissection. E-Journal of Cardiology Practice. 2017;14(38). Disponible en: https://www.escardio.org/Journals/E-Journal-of-Cardiology-Practice/Volume-14/spontaneous-coronary-artery-dissection
15. Tweet MS, Eleid MF, Best PJ, Lennon RJ, Lerman A, Rihal CS, et al. Spontaneous coronary artery dissection: revascularization versus conservative therapy. Circ Cardiovasc Interv. 2014;7:777-86.

16. Alfonso $F$, Paulo M, Gonzalo N, Dutary J, Jiménez-Quevedo $P$, Lennie $\mathrm{V}$, et al. Diagnosis of spontaneous coronary artery dissection by optical coherence tomography. J Am Coll Cardiol. 2012;59:1073-9.

17. Saw J. Spontaneous coronary artery dissection. Can J Cardiol. 2013:29:1027-33.

18. Alfonso F, Bastante T, Rivero F, Cuesta J, Benedicto A, Saw J, et al. Spontaneous coronary artery dissection. Circ J. 2014;78:2099-110.

19. Lempereur M, Fung A, Saw J. Stent malapposition with resorption of intramural hematoma with spontaneous coronary artery dissection. Cardiovasc Diagn Ther. 2015;5:323-9. 